\title{
Guest Editorial Special Issue on Bioelectronic Circuits for Enhanced Diagnosis and Therapy
}

$\mathrm{T}$ HE APPLICATION of innovative circuits and systems in healthcare is experiencing remarkable growth worldwide, spanning from systems supporting health monitoring to rehabilitation. Examples include electronics for smart biosensors to detect or monitor the progress of various diseases, implantable neural prostheses to replace lost function due to neural damage, wireless brain-machine interfaces for neuroscience research and control of prosthetic devices, and new generation cardiovascular technology with real time monitoring capability. Advances in medical device technology have been shown to greatly improve the quality of life for people with conditions such as Parkinson's dis ease, diabetes, hearing loss, blindness and heart failure, to name a few. The purpose of this special is sue is to report the cutting-edge development of circuits and systems which have the potential to enhance diagnosis and therapy.

We received a large number of submissions in response to our call for papers, almost 100 manuscripts, of which 22 papers have been finally accepted for publication in this Special Issue of IEEE TRANSACTIONS ON CIRCUITS AND SYSTEMS-II: EXPRESS BRIEFS (TCAS-II). All the papers have been reviewed by at least three independent reviewers and were selected for publication based on the timeliness and fit of the topic, their technical and tutorial quality and their ability to demonstrate advancement of the state-of-the-art with measured results or (as an exception) with detailed simulated results.

The short length of the individual papers enables us to provide a broad (although naturally incomplete) view of the area of circuits and systems focusing on diverse aspects in enhanced diagnosis and therapy. The papers describe latest developments in multichannel signal processing, wireless power transfer through the human body, tissue sensing, neural stimulation, lab-on-a-chip, high frequency circuits including ultra-wide band (UWB) for cancer detection as well as ultra low-power circuits.

- The contributions Quantization Effects in an Analog-to-Information Front-end in EEG TeleMonitoring, A Flexible Multi-channel EEG Feature Extractor and Classifier for Seizure Detection, and $A$
Fully-Integrated IC with 0.85- $\mu W /$ Channel Consumption for Epileptic iEEG Detection focus on systems for processing the electroencephalogram (EEG) in order to extract information from the raw signal.

- The papers Optimization of the Power Transfer Through Human Body with an Auto Tuning System Using a Synchronous Switched Capacitor, High Efficiency Fully CMOS VCO Rectifier for $\mu$ Watt Resonant Wireless Power Transfer, and Implant Position Estimation via Wireless Power Link highlight aspects of power transfer to implants using inductive coupling.

- $\quad$ Direct RF Subsampling Receivers Enabling Impulse Based UWB Signals for Breast Cancer Detection, A 180 Vpp Integrated Linear Amplifier for Ultrasonic Imaging Applications in a High-Voltage CMOS SOI Technology, are concerned with high frequency circuits targeting diagnostic applications.

- $\quad A$ Wideband Low Distortion CMOS Current Driver for Tissue Impedance Analysis, A Capacitanceto-Frequency Converter with On-chip Passivated Microelectrodes for Bacteria Detection in Saline Buffers up to $575 \mathrm{MHz}$ and The Bio-Oscillator: $A$ Circuit for Cell-Culture Assays present work in the area of tis sue impedance spectros copy.

- A Feasibility Study on the Adoption of Human Body Communication for Medical Service, Towards an Ultra Low Power On-board Processor for Tongue Drive System, Efficient Implementation and Design of A New Single-Channel Electrooculographybased Human-Machine Interface System, describe applications in the broader category of diagnosis and therapy.

- $\quad$ Finally, the papers A HV-CMOS Integrated Circuit for Neural Stimulation in Prosthetic Applications, and A Sub-micro Watt Implantable Capacitive Sensor System for Biomedical Applications, describe implantable circuits for neural stimulation and capacitive sensing respectively.

We would like to thank all the authors, the reviewers who participated in the evaluation of the papers, and the TCAS-II Editorial Staff for their contributions to creating this Special Issue. We hope that this is sue will provide new insights and a glimpse 
of the latest advances within the broad spectrum encompassing bioelectronic circuits.

ROBERT RIEGER

National Sun Yat-Sen University

Department of Electrical Engineering

804, Kaohsiung

Taiwan

rrieger@mail.nsysu.edu.tw

JASWINDER LOT A

University of East London

School of Architecture Computing and Engineering

Docklands Campus, London E16 2RD

United Kingdom

j.lota@uel.ac.uk

XIAO LIU

University College London

Department of Electronic and Electrical Engineering

Torrington Place

London WC1E 7JE

United Kingdom

xiao@ucl.ac.uk 
